\title{
CT quantification of the heterogeneity of fibrosis boundaries in idiopathic pulmonary fibrosis
}

\author{
Junghoan Park ${ }^{1,2}$. Julip Jung ${ }^{3}$ Soon Ho Yoon ${ }^{1,2}$ (D) Helen Hong ${ }^{3} \cdot$ Hyungjin Kim ${ }^{1,2} \cdot$ Heekyung Kim $^{4}$. \\ Jeong-Hwa Yoon ${ }^{5}$. Jin Mo Goo ${ }^{1,2,6}$
}

Received: 9 July 2020 / Revised: 29 October 2020 / Accepted: 2 December 2020 / Published online: 13 January 2021

(C) European Society of Radiology 2021

\begin{abstract}
Objectives To quantify the heterogeneity of fibrosis boundaries in idiopathic pulmonary fibrosis (IPF) using the Gaussian curvature analysis for evaluating disease severity and predicting survival.

Methods We retrospectively included 104 IPF patients and 52 controls who underwent baseline chest CT scans. Normal lungs below - 500 HU were segmented, and the boundary was three-dimensionally reconstructed using in-house software. Gaussian curvature analysis provided histogram features on the heterogeneity of the fibrosis boundary. We analyzed the correlations between histogram features and the gender-age-physiology (GAP) and CT fibrosis scores. We built a regression model to predict diffusing capacity of carbon monoxide (DLCO) using the histogram features and calculated the modified GAP (mGAP) score by replacing DLCO with the predicted DLCO. The performances of the GAP, CT-GAP, and mGAP scores were compared using 100 repeated random-split sets.

Results Patients with moderate-to-severe IPF had more numerous Gaussian curvatures at the fibrosis boundary, lower uniformity, and lower 10th to 30th percentiles of Gaussian curvature than controls or patients with mild IPF (all $p<0.0033$ ). The 20th percentile was most significantly correlated with the GAP score $(r=-0.357 ; p<0.001)$ and the CT fibrosis score $(r=-0.343$; $p=0.001)$. More numerous Gaussian curvatures, higher entropy, lower uniformity, and 10th to 30th percentiles $(p<0.001-0.041)$ were associated with mortality. The mGAP score was comparable to the GAP and CT-GAP scores for survival prediction (mean C-indices, 0.76 vs. 0.79 vs. 0.77 , respectively).

Conclusions Gaussian curvatures of fibrosis boundaries became more heterogeneous as the disease progressed, and heterogeneity was negatively associated with survival in IPF.

Key Points

- Gaussian curvature of the fibrotic lung boundary was more heterogeneous in patients with moderate-to-severe IPF than those with mild IPF or normal controls.

- The 20th percentile of the Gaussian curvature of the fibrosis boundary was linearly correlated with the GAP score and the CT fibrosis score.

- A modified GAP score that replaced the diffusing capacity of carbon monoxide with a composite measure using histogram features of the Gaussian curvature of the fibrosis boundary showed a comparable ability to predict survival to both the GAP and the CT-GAP score.
\end{abstract}

Keywords Tomography, X-ray computed · Lung · Idiopathic pulmonary fibrosis · Quantitative evaluation

Soon Ho Yoon

yshoka@gmail.com

1 Department of Radiology, Seoul National University Hospital, 101 Daehak-ro, Jongno-gu, Seoul 03080, Republic of Korea

2 Department of Radiology, Seoul National University College of Medicine, Seoul, Republic of Korea
3 Department of Software Convergence, Seoul Women's University, Seoul, Republic of Korea

4 Deparment of Radiology, Eulji Medical Center, Eulji University School of Medicine, Daejeon, Republic of Korea

5 Interdisciplinary Program in Medical Informatics, Seoul National University College of Medicine, Seoul, Republic of Korea

6 Institute of Radiation Medicine, Seoul National University Medical Research Center, Seoul, Republic of Korea 


$\begin{array}{ll}\text { Abbreviations } \\ \text { CI } & \text { Confidence interval } \\ \text { DLCO } & \text { Diffusing capacity of carbon monoxide } \\ \text { DLCO } \% & \begin{array}{l}\text { Percent of predicted diffusing capacity of carbon } \\ \text { monoxide }\end{array} \\ \text { FVC } & \text { Forced vital capacity } \\ \text { FVC\% } & \text { Percent of predicted forced vital capacity } \\ \text { GAP } & \text { Gender-age-physiology } \\ \text { GGO } & \text { Ground-glass opacity } \\ \text { IPF } & \text { Idiopathic pulmonary fibrosis } \\ \text { LASSO } & \text { Least absolute shrinkage and selection operator } \\ \text { mGAP } & \text { Modified gender-age-physiology } \\ \text { PFT } & \text { Pulmonary function test } \\ \text { RMSE } & \text { Root mean square error }\end{array}$

\section{Introduction}

Idiopathic pulmonary fibrosis (IPF) is a chronic, progressive, fibrosing interstitial lung disease of unknown etiology [1,2]. IPF patients typically have a median survival of about 3 years after diagnosis. Nevertheless, disease severity may vary considerably at the time of IPF diagnosis, and patients' clinical course after diagnosis can likewise show variation [3]. The gender-age-physiology (GAP) model is a well-validated clinical model for staging disease severity and predicting survival in IPF patients; this model consists of four clinical variables, including age, sex, forced vital capacity (FVC), and diffusing capacity of carbon monoxide (DLCO) $[4,5]$.

The radiologic abnormalities of IPF on chest CT can be utilized not only for diagnosis $[1,6]$, but also for staging its severity and prognostication. The visually assessed extent of fibrosis on CT images is a predictor of survival in IPF patients [7-10]. Furthermore, the CT-derived extent of fibrosis can be used as a supplement to the GAP score to improve the capability of predicting survival [11], or as a substitute for DLCO in the GAP model [12]. However, the visual evaluation of fibrosis on $\mathrm{CT}$ can be limited by the subjective interpretation or degree of experience of the reader, causing inter-observer variation [13]. Accordingly, several computer-based algorithms for the quantification of fibrosis have been developed [14-16] and shown to predict survival or disease progression in IPF better than visual assessments [17-21].

Pre-existing techniques for visual assessment or automatic quantification have focused on the presence and degree of fibrosis [22]. The temporal and spatial heterogeneity of interstitial fibrosis is considered a pathognomonic histologic finding of IPF [23]. Gaussian curvature is defined as the product of the minimum and maximum curvatures (i.e., the reciprocal of the radius) and is one of the local geometric descriptors of surface roughness in classical differential geometry (Fig. 1a and Supplementary figure 1) [24], which has been applied to analyze curvature in the developing brain using brain MRI
[25]. We hypothesized that the similar approach could be applied for IPF patients by quantifying the heterogeneity of the fibrosis. Thus, the purpose of our study was to quantify the spatial heterogeneity of the fibrosis boundary on chest CT in IPF using the Gaussian curvature analysis for evaluating disease severity and predicting survival.

\section{Materials and methods}

Our institutional review board approved this retrospective study (IRB No. H-1803-120-932), and informed consent was waived.

\section{Patients}

A study coordinator initially searched electronic medical records to identify patients who were diagnosed with IPF and underwent chest CT scans from 2006 to 2016 at a single tertiary hospital. The diagnosis of IPF was made according to the $2011 \mathrm{ATS} / \mathrm{ERS} / \mathrm{JRS} / \mathrm{ALAT}$ guideline since 2012 [26]. IPF was diagnosed by surgical pathology until 2011. We applied the following inclusion criteria to determine eligibility: (a) available baseline standard dose thin-section chest CT scans with a slice interval of $1.5 \mathrm{~mm}$ or shorter, and (b) available baseline pulmonary function test (PFT) results within 6 months before or after the baseline chest CT scan. We excluded patients who had (a) malignancy at the baseline workup, (b) a destroyed lung due to other pathology, or (c) profound motion artifacts on their CT images. Finally, a total of 104 patients (mean age, 68.2 \pm 9.4 ; male-to-female ratio, 71:33; 56 diagnosed by pathology, 12 by multidisciplinary diagnosis, and 36 by typical usual interstitial pneumonia CT findings after excluding other differential diagnoses) were included in this study (Fig. 2).

For normal controls, we searched consecutive subjects who underwent chest CT scans from January 1 to April 15, 2019, and had few respiratory symptoms and CT abnormalities except for small benign nodules or linear atelectasis. We applied the same inclusion criteria except for PFT, as PFT was rarely performed for the normal controls without respiratory complaints. After age- and sex-matching with the IPF patients, 52 control subjects (mean age, $67.5 \pm 9.4$; male-to-female ratio, 35:17) were included in this study (Fig. 2).

\section{Clinical risk assessment and CT visual analysis}

The GAP stage and score were calculated using the baseline PFT results, including the percent of predicted FVC (FVC\%) and DLCO (DLCO\%) [4], with the following distribution: GAP stage I, 64 patients; stage II, 34 patients; stage III, 5 patients. Since there were few GAP stage III patients, we classified the patients as having mild IPF (GAP stage I, $n=$ 
a

Normal vector of $\mathrm{P}$

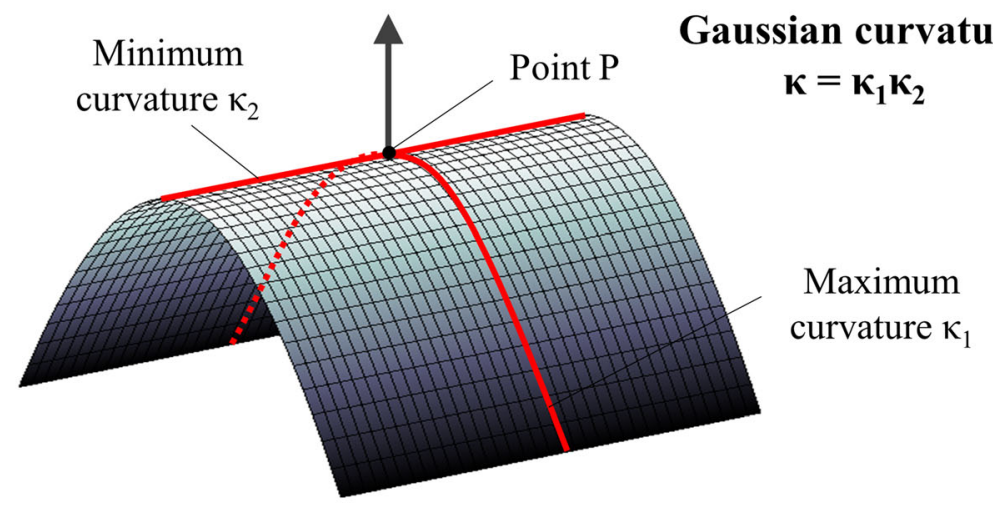

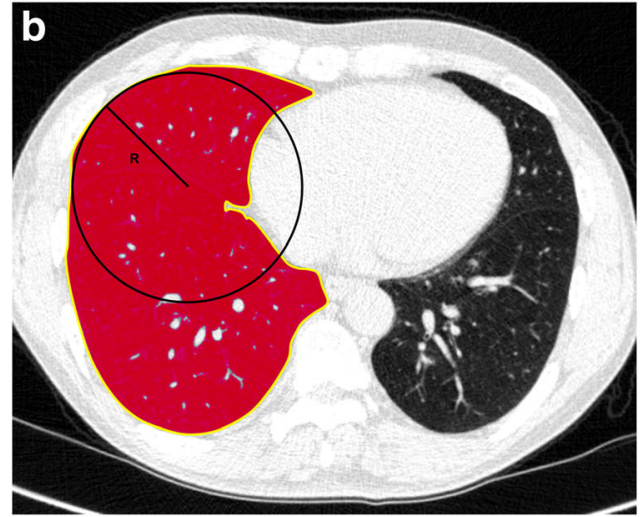

Fig. 1 Schematic diagram of (a) Gaussian curvature of the fibrosis boundaries of (b) the normal lung and (c) the fibrotic lung. a Gaussian curvature is defined as the product of two principal curvatures which are the maximum $\left(\kappa_{1}\right)$ and minimum $\left(\kappa_{2}\right)$ of curvatures that intersect the normal plane - a plane containing the normal vector of the surface at a certain point (P). b In the normal lung, fibrotic areas do not exist; therefore, the boundary (colored in yellow) of non-fibrotic areas (below -500

64) or moderate-to-severe IPF (GAP stage II-III, $n=40$ ). The mean interval between the baseline PFT and the CT scans was $21.2 \pm 38.2$ days.

Two thoracic radiologists (with 13 and 6 years of thoracic CT experience, respectively) blinded to the clinical data analyzed the CT images visually by a

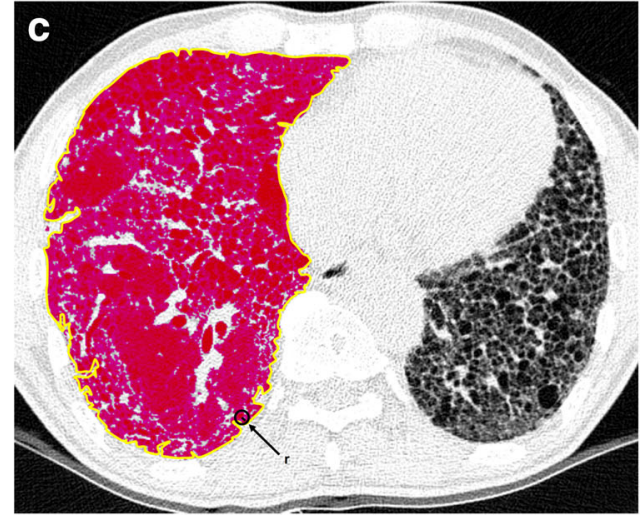

HU is colored in red) corresponds to pleural surfaces, which are smoothly elliptic and have a radius of the tangent sphere (R) that is mostly larger than $1 \mathrm{~mm}$. c In IPF, the fibrosis boundary (colored in yellow) is irregular and the radius of the tangent sphere (r) is much smaller. Thus, the frequency of either positive or negative Gaussian curvature (according to the direction of the boundary curvature) is higher, although overall concavity of the boundary is maintained along pleural surfaces

consensus reading. The radiologists reviewed axial CT images containing lung parenchyma at a PACS workstation (INFINITT PACS Version 5.0.0) with a lung window setting (level, - $700 \mathrm{HU}$; width, $1500 \mathrm{HU}$ ). A whole-lung extent of ground-glass opacity (GGO), consolidation, reticular abnormality, honeycombing,
Fig. 2 Study diagram for the inclusion of patients and controls

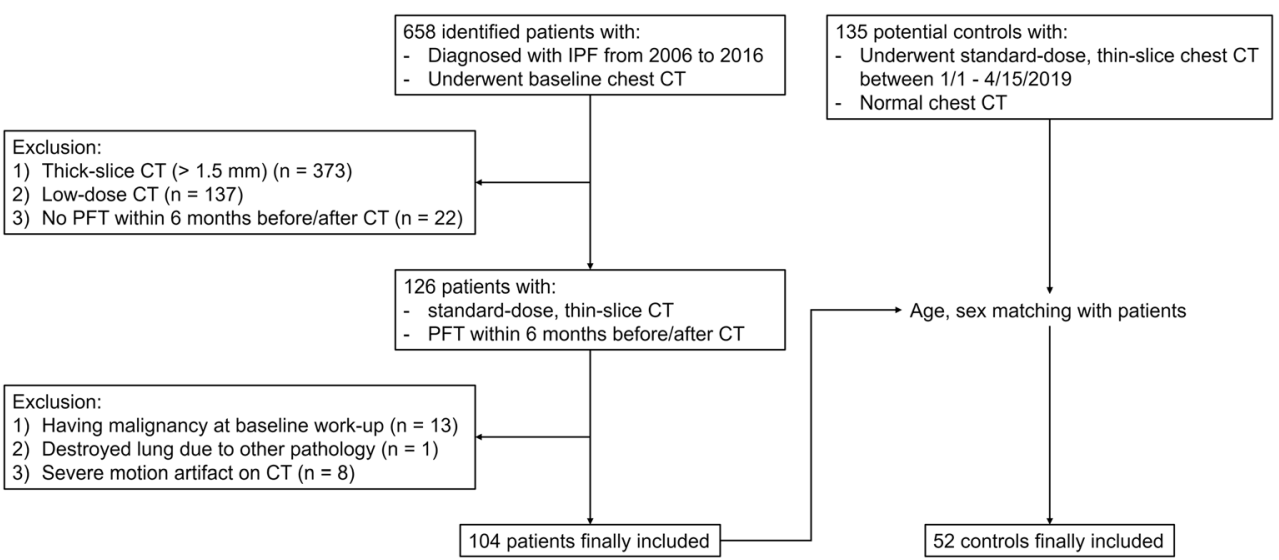


emphysema, and total fibrosis (including both reticular abnormality and honeycombing) were scored to the nearest $10 \%$ [27].

\section{Gaussian curvature histogram analysis of the fibrosis boundaries}

Gaussian curvature analysis of the fibrosis boundaries was performed by one researcher using in-house software (Supplementary figure 2). After loading the $\mathrm{CT}$ images, the non-fibrotic areas of both lungs were segmented with a threshold value of $-500 \mathrm{HU}$ [28]. Next, the subpleural boundary between the fibrotic and non-fibrotic areas was automatically reconstructed by using the marching cube algorithm [29], which is one of the most commonly used algorithms for reconstructing a polygonal mesh from three-dimensional medical data (Fig. 3). This algorithm was performed after the volumetric segmentation of the structure of interest, and by "marching" through all the cubic cells intersected by the isosurface, the algorithm calculated the intersection points along the edges and produced a triangular mesh to fit the points. Finally, Gaussian curvature histogram analysis was performed to provide the following histogram parameters of the Gaussian curvature of the fibrosis boundary [24]: mean, standard deviation, skewness, kurtosis, entropy, uniformity, minimum, maximum, and 10th to 90th percentile values [30].
Fig. 3 Representative images of extracting the boundaries between fibrotic and non-fibrotic lung areas. a, d Baseline chest CT images of patients with (a) mild IPF (GAP stage I) and (d) severe IPF (GAP stage III). b, e After segmenting into fibrotic and nonfibrotic areas using a threshold value of $-500 \mathrm{HU}$, the fibrosis boundary was automatically extracted and reconstructed threedimensionally by applying marching cube. c, f The Gaussian curvature analysis was performed at the fibrosis boundary. The Gaussian curvature value at each point of the boundary was coded into red (negative), green (nearzero), and blue (positive). Note that the fibrosis boundary in severe IPF is more irregular than in mild IPF
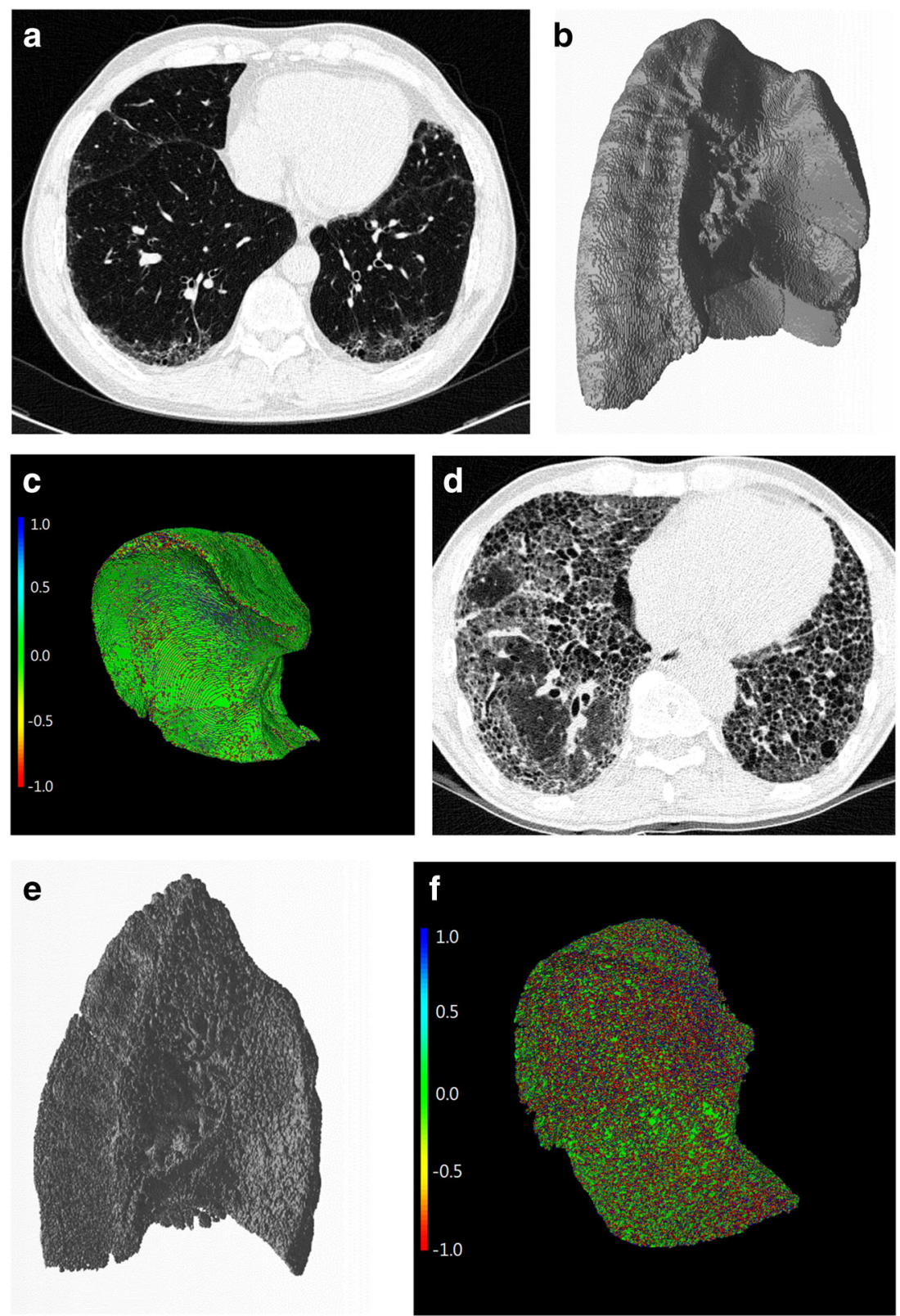


\section{Survival analysis}

All patients were followed up until December 31, 2017. We obtained survival data regarding whether the patients were alive until December 31, 2017, and the date of death if the patients died earlier from the Ministry of the Interior and Safety of South Korea. The mean follow-up period was 47.8 \pm 35.2 months.

\section{Prediction of DLCO using the CT histogram parameters of Gaussian curvature}

To predict DLCO using the histogram parameters, the least absolute shrinkage and selection operator (LASSO) was used to select features and to build a regression model [31]. First, study participants were split randomly into training and validation sets (6:4). Then, a linear regression model for predicting DLCO was built using the training set, and the tuning parameter for model shrinkage (i.e., lambda) was obtained based on the four-fold cross-validation error. The model was assessed on the validation set.

\section{Statistical analysis}

One-way ANOVA was used to compare the histogram parameters between IPF patients and controls. The Tukey honest significant difference test was used as a post hoc test. The Pearson or Spearman rank correlation coefficient was used to analyze the correlations of the histogram parameters with the GAP score and CT visual analysis score. A Cox proportional hazard model was used to investigate the effects of the histogram parameters, CT visual analysis score, and clinical parameters on patient survival. A $p<0.05$ was selected to determine statistical significance, and the Bonferroni correction was applied for comparison of the histogram parameters between IPF patients and controls.

The DLCO prediction model was evaluated with respect to the following metrics: (1) root mean square error (RMSE) and (2) $R$-square. The modified GAP (mGAP) score was calculated by replacing DLCO\% with the predicted DLCO\% in the GAP score formula. We compared the $\mathrm{C}$-indices and their $95 \%$ confidence intervals (CIs) of the GAP, CT-GAP, and mGAP scores using 100-times repeated random splitting of the study population [32]. The comparison was considered to be significant when $p<0.05$.

Statistical analyses were carried out using the SPSS software (version 25; IBM Corp.) and R software (version 3.5.1, $\mathrm{R}$ Project for Statistical Computing; packages: caret and glmnet).

\section{Results}

\section{Baseline characteristics and CT visual analysis}

The patients with moderate-to-severe IPF were significantly older ( $71.4 \pm 7.8$ vs. $66.2 \pm 9.8$ years, $p=0.014)$ and had lower FVC\% $(63.4 \pm 11.4 \%$ vs. $83.4 \pm 14.8 \%, p<0.001)$ and DLCO \% (49.4 $\pm 18.1 \%$ vs. $69.9 \pm 16.4 \%, p<0.001)$ than those with mild IPF. Furthermore, patients with moderate-tosevere IPF had a larger extent of reticular opacities and honeycombing, as well as a higher CT fibrosis score $(p<$ 0.001-0.002) than patients with mild IPF, but the extent of GGO, consolidation, and emphysema did not significantly differ between the groups (Table 1).

\section{Quantitative analysis of the heterogeneity of fibrosis boundaries}

Among the histogram parameters of the Gaussian curvature, the overall number, mean, entropy, uniformity, and the 10th to 30th percentiles were significantly different across controls, patients with mild IPF, and those with moderate-to-severe IPF $(p<0.0033)$ (Table 2, Supplementary table 1, and Figs. 4 and $5)$. In the post hoc test, the 10th and 20th percentiles were lower in patients with mild IPF than in controls $(p<0.001-$ 0.002 ), and those values were also lower in patients with moderate-to-severe IPF than in patients with mild IPF ( $p \leq$ 0.001). Patients with moderate-to-severe IPF had a significantly larger number of curvatures, higher entropy and lower uniformity, and 30th percentile than controls $(p<0.001)$ and larger number of curvatures, lower uniformity, and 30th percentile than patients with mild IPF $(p<0.001-0.003)$, but there was no significant difference of those parameters between controls and patients with mild IPF. Histogram parameters of the Gaussian curvature did not significantly differ depending on contrast enhancement (Supplementary table 2).

\section{Association between the GAP score, CT visual analysis, and heterogeneity of fibrosis boundaries}

The overall number of the curvatures and entropy showed significant positive correlations with the GAP score $(r=$ $0.308, p=0.002 ; r=0.200, p=0.043$, respectively), while uniformity and the 10th to 30 th percentiles showed significant negative correlations with the GAP score $(r$ ranging from 0.357 to $-0.219, p<0.05$ ). The 20th percentile of Gaussian curvature showed the most significant correlation with the GAP score $(r=-0.357 ; p<0.001)$. However, histogram parameters did not have a significant correlation with the CT-GAP score except for the overall number of curvatures $(r=0.236, p=0.016)$ (Table 3 and Supplementary table 3$)$.

The 20th percentile showed the most significant correlation with the CT fibrosis score $(r=-0.343 ; p=0.001)$ and the 
Table 1 Summary of clinical parameters and CT visual analysis scores

\begin{tabular}{|c|c|c|c|c|}
\hline Variables & Control $(n=52)$ & Mild IPF $(n=64)$ & Moderate-to-severe IPF $(n=40)$ & $p$ value $^{\dagger}$ \\
\hline \multicolumn{5}{|l|}{ Clinical parameters } \\
\hline Age (years) & $67.5 \pm 9.4$ & $66.2 \pm 9.8$ & $71.4 \pm 7.8$ & $0.018^{* \mathrm{a}}$ \\
\hline Male sex ${ }^{\mathrm{b}}$ & $35(67.3 \%)$ & $40(62.5 \%)$ & $31(77.5 \%)$ & 0.278 \\
\hline FVC (L) & - & $2.74 \pm 0.76$ & $2.09 \pm 0.57$ & $<0.001 *$ \\
\hline DLCO (mL/mmHg/min) & - & $12.04 \pm 3.28$ & $7.72 \pm 2.46^{\mathrm{c}}$ & $<0.001 *$ \\
\hline $\mathrm{FVC} \%$ & - & $83.4 \pm 14.8$ & $63.4 \pm 11.4$ & $<0.001 *$ \\
\hline DLCO $\%$ & - & $69.9 \pm 16.4$ & $49.4 \pm 18.1^{\mathrm{c}}$ & $<0.001 *$ \\
\hline Unable to perform DLCO test ${ }^{\mathrm{b}}$ & - & $0(0.0 \%)$ & $1(2.5 \%)$ & 0.385 \\
\hline \multicolumn{5}{|l|}{$\mathrm{CT}$ visual analysis score (\%) } \\
\hline GGO & - & $11.1 \pm 9.3$ & $13.5 \pm 8.6$ & 0.190 \\
\hline Consolidation & - & $3.0 \pm 6.1$ & $4.8 \pm 7.8$ & 0.225 \\
\hline Reticular abnormality & - & $27.8 \pm 7.2$ & $32.5 \pm 6.7$ & $0.001 *$ \\
\hline Honeycombing & - & $13.0 \pm 10.5$ & $21.3 \pm 13.8$ & $0.002 *$ \\
\hline Total fibrosis (CT fibrosis score) & - & $32.0 \pm 8.8$ & $41.8 \pm 10.1$ & $<0.001 *$ \\
\hline Emphysema & - & $6.3 \pm 8.6$ & $10.3 \pm 12.1$ & 0.073 \\
\hline
\end{tabular}

Unless otherwise indicated, data are mean \pm standard deviation. Asterisks indicate statistical significance $(p<0.05)$

$I P F$ idiopathic pulmonary fibrosis, $F V C$ forced vital capacity, $D L C O$ diffusing capacity of carbon monoxide, $F V C \%$ percent of predicted FVC, $D L C O \%$ percent of predicted DLCO, GGO ground-glass opacity

${ }^{\dagger} p$ values were obtained using one-way ANOVA for age, chi-square test for "male sex," Fisher's exact test for "unable to perform DLCO test," and Student's $T$ test for other variables

${ }^{a}$ Post hoc test for age: $p=0.733$ (normal control vs. mild IPF), $p=0.104$ (normal control vs. moderate-to-severe IPF), and $p=0.014$ (mild IPF vs. moderate-to-severe IPF)

${ }^{\mathrm{b}}$ Data are absolute numbers, with percentages in parentheses

${ }^{\mathrm{c}}$ One patient who was unable to perform the DLCO test was excluded

extent of honeycombing on CT $(r=-0.362 ; p<0.001)$. A larger number of the curvatures, smaller minimum or 10th to 30 th percentile values, and larger maximum or 90th percentile values were associated with a greater extent of at least one lung abnormality, including consolidation, reticular abnormality, honeycombing, and total fibrosis (Table 4 and Supplementary table 4). However, none of the histogram parameters displayed significant correlations with the extent of GGO or emphysema.

\section{Survival analysis with the prediction of DLCO using the histogram features of Gaussian curvature}

There were $56(53.8 \%)$ deaths among the IPF patients during follow-up. The 1-, 2- and 3-year survival rates were $87 \%, 74 \%$, and $61 \%$, respectively. In univariate survival analysis, a higher GAP score $(p<0.001)$, as well as the clinical parameters included in the GAP score - older age, male sex, lower $\mathrm{FVC} \%$, and DLCO $\%(p<0.001-0.007)$ - were associated with worse survival. Among the $\mathrm{CT}$ visual analysis parameters, a greater extent of reticular abnormality and honeycombing, the total fibrosis score, and emphysema were associated with worse survival $(p<0.001)$, while GGO and consolidation were not associated with survival. Among the histogram parameters, the overall number of the curvatures $(p<0.001)$, higher entropy ( $p=0.041)$, lower uniformity $(p=0.027)$, and lower 10th to 30th percentiles $(p<0.001-0.010)$ were significant survival predictors (Table 5).

The regression equation for the prediction of DLCO\% based on a single random split was as follows:

Predicted DLCO $\%=74.456+18.227 *$ mean $+33.553 * 20$ th percentile $-0.003 *$ maximum

The median values of DLCO\% and predicted DLCO \% of the 100 repeated random-split set were $60 \%$ (interquartile range, $48-74 \%$ ) and $63 \%$ (interquartile range, $57-67 \%$ ), respectively. The mean $R$-square value between DLCO $\%$ and predicted DLCO \% was 0.296 (95\% CI, 0.275-0.317), and the RMSE was 16.69 (95\% CI, 16.40-16.97). The predicted DLCO\% was significantly associated with patient survival in univariate survival analysis using validation set (hazard ratio, $0.905 ; 95 \% \mathrm{CI}, 0.845-0.969 ; p=0.004$ based on a single random split), and the mGAP score with the predicted DLCO $\%$ was also significantly associated with worse survival (hazard ratio, 34.0; 95\% CI, 7.0-165.7; $p<0.001$ based on a single random split). 
Table 2 Summary of comparison of the histogram parameters of the Gaussian curvature of fibrosis boundaries

\begin{tabular}{|c|c|c|c|c|c|c|c|}
\hline \multirow[t]{2}{*}{ Histogram parameters } & \multirow[t]{2}{*}{ Control $(n=52)$} & \multirow[t]{2}{*}{ Mild IPF $(n=64)$} & \multirow{2}{*}{$\begin{array}{l}\text { Moderate-to- } \\
\text { severe IPF }(n=40)\end{array}$} & \multirow[t]{2}{*}{$p$ value $^{\dagger}$} & \multicolumn{3}{|c|}{$p$ value for post hoc test ${ }^{\dagger \dagger}$} \\
\hline & & & & & $\begin{array}{l}\text { Control vs. } \\
\text { mild IPF }\end{array}$ & $\begin{array}{l}\text { Control vs. moderate- } \\
\text { to-severe IPF }\end{array}$ & $\begin{array}{l}\text { Mild vs. moderate- } \\
\text { to-severe IPF }\end{array}$ \\
\hline $\begin{array}{l}\text { Overall no. of } \\
\quad \text { curvatures }\left(\times 10^{6}\right)\end{array}$ & $1.587 \pm 0.342$ & $1.799 \pm 0.593$ & $2.429 \pm 1.030$ & $<0.001 *$ & 0.215 & $<0.001 *$ & $<0.001 *$ \\
\hline Mean & $0.700 \pm 0.263$ & $0.545 \pm 0.211$ & $0.575 \pm 0.201$ & $0.001 *$ & $0.001 *$ & 0.027 & 0.792 \\
\hline Entropy & $1.549 \pm 0.317$ & $1.620 \pm 0.347$ & $1.831 \pm 0.317$ & $<0.001 *$ & 0.480 & $<0.001 *$ & 0.005 \\
\hline Uniformity & $0.572 \pm 0.083$ & $0.550 \pm 0.086$ & $0.494 \pm 0.077$ & $<0.001 *$ & 0.325 & $<0.001 *$ & $0.003 *$ \\
\hline 10th percentile & $-0.995 \pm 0.326$ & $-1.243 \pm 0.405$ & $-1.531 \pm 0.417$ & $<0.001 *$ & $0.002 *$ & $<0.001 *$ & $0.001 *$ \\
\hline 20th percentile & $-0.410 \pm 0.197$ & $-0.589 \pm 0.226$ & $-0.792 \pm 0.261$ & $<0.001 *$ & $<0.001 *$ & $<0.001 *$ & $<0.001 *$ \\
\hline 30th percentile & $-0.036 \pm 0.122$ & $-0.130 \pm 0.162$ & $-0.278 \pm 0.210$ & $<0.001 *$ & 0.008 & $<0.001^{*}$ & $<0.001 *$ \\
\hline $\begin{array}{l}\text { Other } \\
\text { parameters }\end{array}$ & - & - & - & N.S. & N.S. & N.S. & N.S. \\
\hline
\end{tabular}

Data are mean \pm standard deviation. Asterisks indicate statistical significance ( $p<0.0033$, applying the Bonferroni correction). Complete data are available in Supplementary table 1

$I P F$ idiopathic pulmonary fibrosis, N.S. not significant

${ }^{\dagger} p$ values were obtained using one-way ANOVA

${ }^{\dagger} p$ values were obtained using the Tukey honest significant difference test
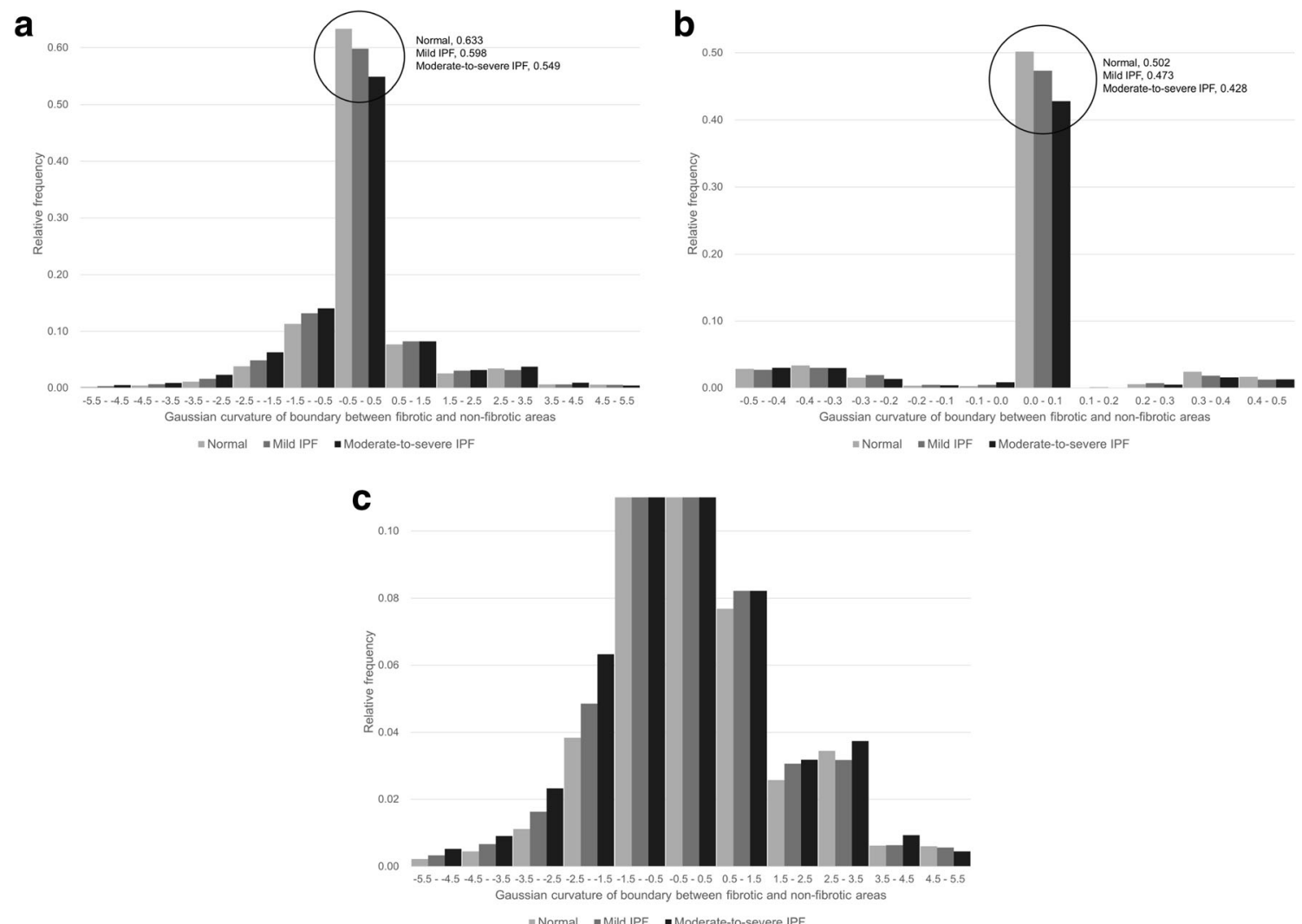

Fig. 4 Summary histogram of the Gaussian curvature of the fibrosis boundaries in healthy controls and patients with mild and moderate-tosevere IPF. a, b The general shape of the histogram of the Gaussian curvature of the fibrosis boundaries is similar across groups, as the overall concavity of the boundaries is maintained along pleural surfaces,

resulting in a dominant peak of Gaussian curvature at 0 . $\mathbf{c}$ When the upper bound of the relative frequency on the $Y$-axis is adjusted in the same graph, it becomes clear that positively or negatively skewed Gaussian curvatures with a smaller radius exist primarily in IPF patients 

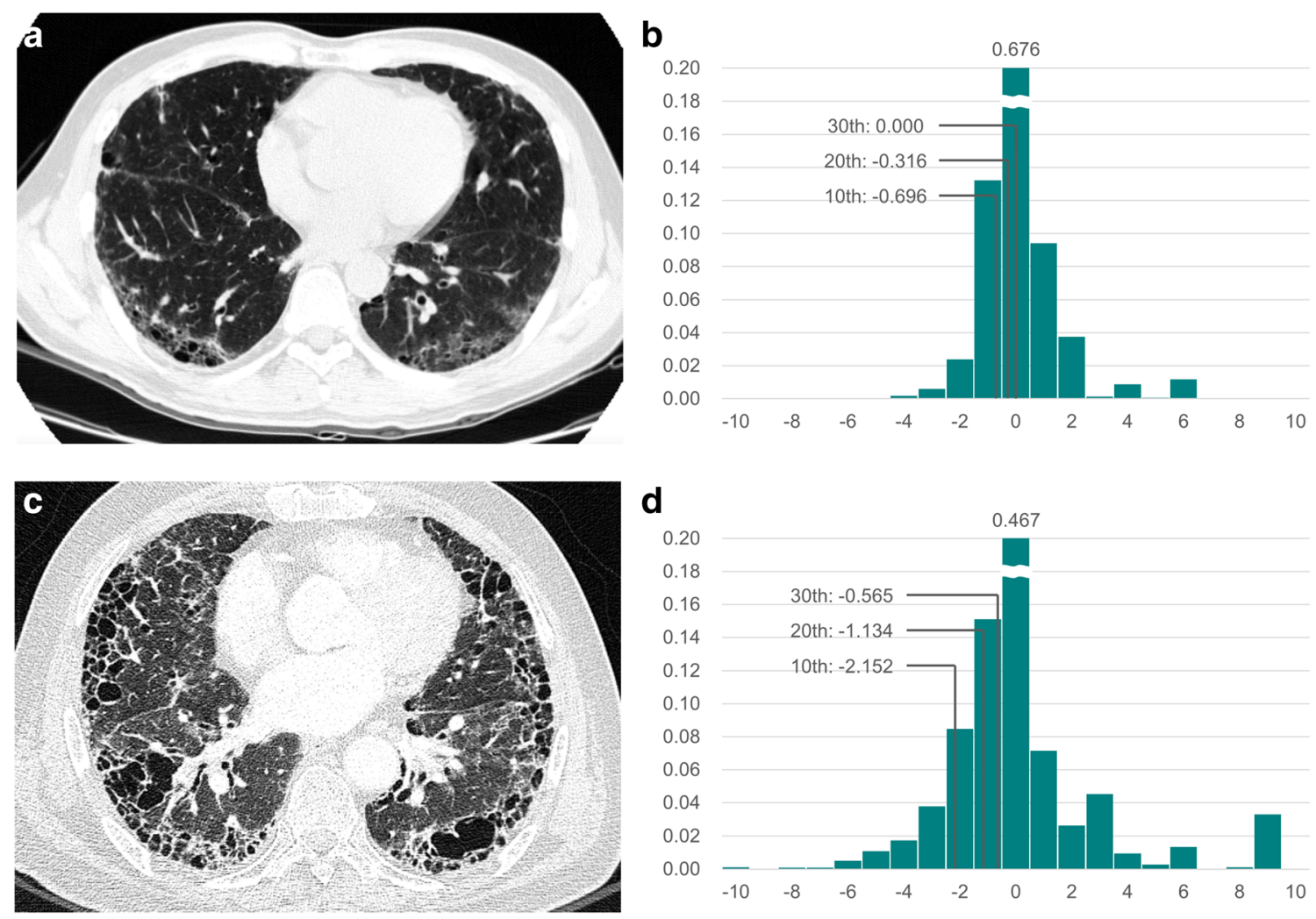

Fig. 5 Representative images of (a, b) mild and (c, d) severe IPF. a Baseline chest CT image of a 50-year-old man with mild IPF (GAP stage I). b The histogram of the Gaussian curvature of the subpleural fibrosis boundary is shown: entropy, 1.141 ; uniformity, 0.664 ; 10 th to 30 th percentiles, $-0.696,-0.316$, and 0.000 , respectively (data with Gaussian curvature $<-10.5$ or $>10.5$ are cropped for visibility). $\mathbf{c}$ Baseline chest

The mean C-indices in the GAP and CT-GAP scores were 0.79 (95\% CI, 0.71-0.86) and 0.77 (95\% CI, 0.68-0.85), respectively. The mGAP score calculated with the predicted

CT image of a 66-year-old man with severe IPF (GAP stage III). $\mathbf{d}$ The histogram of the Gaussian curvature of the subpleural fibrosis boundary is shown: entropy, 2.231; uniformity, 0.400 ; 10 th to 30 th percentiles, $2.152,-1.134$, and -0.565 , respectively (data with Gaussian curvature $<-10.5$ or $>10.5$ are cropped for visibility)

DLCO $\%$ using the composite of the histogram features of the Gaussian curvature instead of DLCO\% had a mean Cindex of 0.76 (95\% CI, 0.69-0.84). All three GAP scores

Table 3 Summary of correlations between the histogram parameters of the Gaussian curvature of fibrosis boundaries and the GAP, CT-GAP, and mGAP scores

\begin{tabular}{|c|c|c|c|c|c|c|}
\hline \multirow[t]{2}{*}{ Histogram parameters } & \multicolumn{2}{|l|}{ GAP score } & \multicolumn{2}{|l|}{ CT-GAP score } & \multicolumn{2}{|l|}{ mGAP score $^{\mathrm{a}}$} \\
\hline & $\begin{array}{l}\text { Pearson's correlation } \\
\text { coefficient }\end{array}$ & $p$ value $^{\dagger}$ & $\begin{array}{l}\text { Pearson's correlation } \\
\text { coefficient }\end{array}$ & $p$ value ${ }^{\dagger}$ & Pearson's correlation coefficient & $p$ value $^{\dagger}$ \\
\hline Overall no. of curvatures & 0.308 & $0.002 *$ & 0.236 & $0.016^{*}$ & 0.341 & $<0.001 *$ \\
\hline Entropy & 0.200 & $0.043 *$ & 0.069 & 0.487 & 0.222 & $0.025 *$ \\
\hline Uniformity & -0.219 & $0.026^{*}$ & -0.083 & 0.404 & -0.235 & $0.017 *$ \\
\hline 10th percentile & -0.275 & $0.005 *$ & -0.136 & 0.170 & -0.285 & $0.004 *$ \\
\hline 20th percentile & -0.357 & $<0.001 *$ & -0.187 & 0.059 & -0.357 & $<0.001 *$ \\
\hline 30th percentile & -0.321 & $0.001 *$ & -0.182 & 0.066 & -0.326 & $0.001 *$ \\
\hline Other parameters & - & N.S. & - & N.S. & - & N.S. \\
\hline
\end{tabular}

Asterisks indicate statistical significance $(p<0.05)$. Complete data are available in Supplementary table 3

$G A P$ gender-age-physiology, $m G A P$ modified GAP, N.S. not significant

${ }^{\dagger} p$ values were obtained using the Pearson's correlation coefficient

${ }^{a}$ mGAP score was calculated based on a single random split: Predicted DLCO $\%=74.456+18.227 *$ mean $+33.553 * 20$ th percentile $-0.003 *$ maximum 
Table 4 Summary of correlation between the histogram parameters of the Gaussian curvature of fibrosis boundaries and CT visual analysis score

\begin{tabular}{|c|c|c|c|c|}
\hline Variables & Consolidation & Reticular abnormality & Honeycombing & Total fibrosis \\
\hline Overall no. of curvatures & N.S. & N.S. & $0.364\left(<0.001^{*}\right)$ & $0.324(0.001 *)$ \\
\hline Minimum & $-0.204\left(0.038^{*}\right)$ & N.S. & N.S. & $-0.206\left(0.036^{*}\right)$ \\
\hline 10th percentile & $-0.224(0.022 *)$ & N.S. & $-0.286\left(0.003^{*}\right)$ & $-0.309(0.001 *)$ \\
\hline 20th percentile & N.S. & $-0.231(0.019 *)$ & $-0.362(<0.001 *)$ & $-0.343(<0.001 *)$ \\
\hline 30th percentile & N.S. & N.S. & $-0.353(<0.001 *)$ & $-0.313(0.001 *)$ \\
\hline 90th percentile & $0.198\left(0.044^{*}\right)$ & N.S. & N.S. & N.S. \\
\hline Maximum & $0.214(0.029 *)$ & N.S. & N.S. & N.S. \\
\hline Other parameters & N.S. & N.S. & N.S. & N.S. \\
\hline
\end{tabular}

Data are Spearman's $\rho$ coefficients between each histogram parameter and the CT visual analysis score, with $p$ values in parentheses. Asterisks indicate statistical significance $(p<0.05)$. None of the histogram parameters displayed significant correlations with the extent of ground-glass opacity (GGO) or emphysema. Complete data are available in Supplementary table 4

CT computed tomography, N.S. not significant

showed a comparable ability to predict survival without a significant difference in the C-index $(p>0.05 ; 95 \% \mathrm{CI}$ of difference, -0.09 to 0.05 when compared with the GAP score) $(p>0.05 ; 95 \% \mathrm{CI}$ of difference, -0.04 to 0.04 with the CT-GAP score).

\section{Discussion}

Gaussian curvature is positive for elliptic surfaces and negative for saddle surfaces, and the absolute value of Gaussian curvature increases as the radius becomes smaller. In normal lungs, as fibrotic areas do not exist, the boundary of nonfibrotic areas corresponds to the pleural surfaces, which typically have a smooth convexity with some acute peaks between the chest wall pleura and mediastinal or diaphragmatic pleura (Fig. 1b). Therefore, the Gaussian curvature of normal lungs is predominantly distributed around 0 , followed by curvatures larger than 0 . In contrary, IPF typically involves a predominant subpleural pattern of fibrosis, resulting in irregular boundaries between fibrotic and non-fibrotic areas. The irregular boundaries of fibrosis make more numerous Gaussian curvatures and result in many positively or negatively skewed Gaussian curvatures with a smaller radius, which is represented as higher entropy, lower uniformity, lower 10th to 30th percentiles, and higher 70th to 90th percentiles (Fig. 1c).

The overall number and 10th to 30 th percentiles of Gaussian curvatures showed significant correlations with the extent of honeycombing and total fibrosis on CT, and entropy and uniformity also tended to be higher and lower with a greater extent of honeycombing and total fibrosis, respectively. In addition, these parameters were shown to be associated with disease severity and survival in IPF patients. These results are consistent with the aforementioned theoretical relationship between the Gaussian curvature of subpleural surfaces and the extent of fibrosis in IPF. Interestingly, the 70th to 90th percentiles of Gaussian curvature did not show any significant correlations with fibrosis. The 70th to 90th percentiles reflect peaks or pits, whereas the 10th to 30th percentiles reflect saddle points. Considering that even normal lungs have acute peaks at the flexure of the pleura at the transition between the diaphragm and mediastinum, while saddle points are scarce in normal lungs, the 10th to 30th percentiles seem more meaningful.

A previous study by Iwasawa et al reported that the CT analysis of subpleural fibrosis had a prognostic implication in IPF [33]. Despite the similar prognostic implication of subpleural fibrosis, the analyzing methodology of subpleural fibrosis was different between ours and theirs. We analyzed the curvature of fibrosis boundary caused by subpleural fibrosis, and their work analyzed the CT morphology of subpleural fibrosis. The result of the two studies seems to emphasize the importance of preferential subpleural involvement in the disease course of IPF.

The mGAP score calculated with predicted DLCO\% showed a similar ability to predict survival to the GAP and CT-GAP scores. These results imply that the spatial heterogeneity of the fibrosis boundary in IPF can provide similar prognostic information to the degree of fibrosis on CT. The GAP score is a well-validated model for predicting survival in IPF patients, and the CT-GAP model is a useful alternative for the original GAP model [4, 12]. However, the DLCO test can suffer from intra- and inter-session variability, and some patients with lung disease cannot perform the maneuver of the DLCO test adequately [34, 35]. Furthermore, during a regular follow-up, IPF patients typically perform CT scans and spirometry but less undergo a DLCO test. The CT-GAP model allows predicting survival without performing the DLCO test, but visual assessment of the fibrosis on CT requires experienced radiologists and potentially be affected by inter- 
Table 5 Univariate survival analysis for clinical parameters, the CT visual analysis score, and the histogram features of the Gaussian curvature of fibrosis boundaries

\begin{tabular}{|c|c|c|c|}
\hline Variables & Hazard ratio & $95 \% \mathrm{CI}$ & $p$ value $^{\dagger}$ \\
\hline \multicolumn{4}{|l|}{ Clinical parameters } \\
\hline Age (years) & 1.039 & $1.010-1.069$ & $0.007 *$ \\
\hline Male sex & 2.482 & $1.279-4.816$ & $0.007 *$ \\
\hline $\mathrm{FVC} \%$ & 0.962 & $0.944-0.980$ & $<0.001 *$ \\
\hline $\mathrm{DLCO} \%^{\mathrm{a}}$ & 0.974 & $0.959-0.990$ & $0.001 *$ \\
\hline GAP score & 3.722 & $2.467-5.615$ & $<0.001 *$ \\
\hline \multicolumn{4}{|l|}{$\mathrm{CT}$ visual analysis score $(\%)$} \\
\hline Ground-glass opacity & 0.992 & $0.965-1.021$ & 0.597 \\
\hline Consolidation & 1.023 & $0.989-1.059$ & 0.190 \\
\hline Reticular abnormality & 1.083 & $1.040-1.128$ & $<0.001 *$ \\
\hline Honeycombing & 1.050 & $1.029-1.072$ & $<0.001 *$ \\
\hline Total fibrosis & 1.095 & $1.061-1.129$ & $<0.001 *$ \\
\hline Emphysema & 1.047 & $1.022-1.073$ & $<0.001^{*}$ \\
\hline \multicolumn{4}{|l|}{ Histogram features } \\
\hline Overall no. of curvatures $\left(\times 10^{6}\right)$ & 1.605 & $1.230-2.095$ & $<0.001 *$ \\
\hline Mean & 0.894 & $0.237-3.374$ & 0.868 \\
\hline Standard deviation & 1.163 & $0.804-1.682$ & 0.424 \\
\hline Skewness & 0.903 & $0.727-1.123$ & 0.359 \\
\hline Kurtosis & 0.995 & $0.983-1.008$ & 0.478 \\
\hline Entropy & 2.175 & $1.034-4.578$ & $0.041 *$ \\
\hline Uniformity & 0.031 & $0.001-0.667$ & $0.027 *$ \\
\hline Minimum & 0.962 & $0.923-1.004$ & 0.075 \\
\hline 10th percentile & 0.482 & $0.276-0.841$ & $0.010^{*}$ \\
\hline 20th percentile & 0.215 & $0.084-0.554$ & $0.001 *$ \\
\hline 30th percentile & 0.173 & $0.049-0.612$ & $0.007 *$ \\
\hline 70th percentile & 39.493 & $0.058-26919.670$ & 0.269 \\
\hline 80 th percentile & 1.553 & $0.690-3.495$ & 0.287 \\
\hline 90th percentile & 1.212 & $0.874-1.681$ & 0.249 \\
\hline Maximum & 1.000 & $0.960-1.041$ & 0.987 \\
\hline
\end{tabular}

Asterisks indicate statistical significance $(p<0.05)$

$C I$ confidence interval, $F V C \%$ percent-predicted forced vital capacity, $D L C O \%$ percent-predicted diffusing capacity of carbon monoxide, GAP gender-age-physiology.

${ }^{\dagger} p$ values were obtained using a univariate Cox proportional hazard model

${ }^{\text {a }}$ One patient who was unable to perform the DLCO test was excluded observer variability. Either the DLCO test or time-consuming visual grading of the experienced radiologist is limitedly applicable in clinical practice during coronavirus disease 2019 [36]. In our method of analysis, on the other hand, interobserver variability was unlikely to occur since non-fibrotic areas were segmented using a threshold of $-500 \mathrm{HU}$ and the entire process of analysis, from lung segmentation to the Gaussian curvature analysis, was fully automated. A combined analysis of the degree and spatial heterogeneity of fibrosis may provide better prognostication of IPF, and further investigations such as a head-to-head comparison between the GAP scores are warranted.

Our study has several limitations. First, our study was designed retrospectively and included a relatively small population of patients and controls. Second, the CT system and protocols were variable, including both contrast-enhanced and non-enhanced CT, although we excluded low-dose CT scans. Of particular note, CT attenuation of the lung changes depending on the use of intravenous contrast, which may affect the automatic segmentation of the lung using a specified HU threshold [37]. However, the resulting difference would be minimal since the difference between the attenuation of the lung with or without contrast enhancement is much smaller than the difference between fibrotic and non-fibrotic lung tissues [37]. Third, although patients were instructed to achieve full inspiration when undergoing chest $\mathrm{CT}$, the degree of inspiration may have varied among the patients. Insufficient inspiration may increase the CT attenuation of the lung, 
thereby affecting the Gaussian curvature. Fourth, the reproducibility of our analyzing method was not evaluated in this study. Although thought to be minimal, inter-scan variability can occur depending on CT imaging conditions. Fifth, the example of the regression equation mentioned in the result was derived from a single random split, and different parameters may be selected in the other split of the population or external validation. Finally, we analyzed Gaussian curvature of the outmost boundary between fibrotic and non-fibrotic lungs. It predominantly reflected subpleural fibrosis in IPF patients, with a minor exception of the wall of honeycombing cysts which might not be included as a fibrotic area due to smaller HU. Thus, the entire boundary of fibrosis may not have been fully reflected in the analysis. Particularly, a central boundary between fibrotic and non-fibrotic lungs cannot be evaluated with our analyzing method. Further study using the central boundary may be meaningful.

In conclusion, the spatial heterogeneity of the fibrosis boundary in IPF could be quantitatively measured using the Gaussian curvature histogram analysis, and irregular fibrosis boundaries were represented as more numerous curvatures and heterogeneous distribution of Gaussian curvature on histograms. Heterogeneity was negatively associated with disease severity and survival in IPF patients. The quantitative analysis of the spatial heterogeneity of fibrosis boundaries in IPF shows promise as a potential imaging biomarker for staging disease severity and prognostication.

Supplementary Information The online version contains supplementary material available at https://doi.org/10.1007/s00330-020-07594-y.

Funding This study has received funding by the National Research Foundation of Korea (NRF) grant funded by the Korea government (MSIT) (2016R1C1B1015761).

\section{Compliance with ethical standards}

Guarantor The scientific guarantor of this publication is Soon Ho Yoon.

Conflict of interest Soon Ho Yoon is the chief medical officer of MEDICAL IP Co. Ltd. outside this work. Other authors of this manuscript declare no relationships with any companies whose products or services may be related to the subject matter of the article.

Statistics and biometry One of the authors has significant statistical expertise.

Informed consent Written informed consent was waived by the Institutional Review Board.

Ethical approval Institutional Review Board approval was obtained.

\footnotetext{
Methodology

- retrospective

- case-control study/diagnostic or prognostic study

- performed at one institution
}

\section{References}

1. Raghu G, Remy-Jardin M, Myers JL et al (2018) Diagnosis of idiopathic pulmonary fibrosis. An official ATS/ERS/JRS/ALAT clinical practice guideline. Am J Respir Crit Care Med 198:e44-e68

2. Pérez ERF, Daniels CE, Sauver JS et al (2010) Incidence, prevalence, and clinical course of idiopathic pulmonary fibrosis: a population-based study. Chest 137:129-137

3. Kim DS, Collard HR, King TE Jr (2006) Classification and natural history of the idiopathic interstitial pneumonias. Proc Am Thorac Soc 3:285-292

4. Ley B, Ryerson CJ, Vittinghoff E et al (2012) A multidimensional index and staging system for idiopathic pulmonary fibrosis. Ann Intern Med 156:684-691

5. Lee SH, Kim SY, Kim DS et al (2016) Predicting survival of patients with idiopathic pulmonary fibrosis using GAP score: a nationwide cohort study. Respir Res 17:131

6. Kim H, Yoon SH, Hong H, Hahn S, Goo JM (2018) Diagnosis of idiopathic pulmonary fibrosis in a possible usual interstitial pneumonia pattern: a meta-analysis. Sci Rep 8:1-8

7. Best AC, Meng J, Lynch AM et al (2008) Idiopathic pulmonary fibrosis: physiologic tests, quantitative CT indexes, and CT visual scores as predictors of mortality. Radiology 246:935-940

8. Shin KM, Lee KS, Chung MP et al (2008) Prognostic determinants among clinical, thin-section CT, and histopathologic findings for fibrotic idiopathic interstitial pneumonias: tertiary hospital study. Radiology 249:328-337

9. Sumikawa H, Johkoh T, Colby TV et al (2008) Computed tomography findings in pathological usual interstitial pneumonia: relationship to survival. Am J Respir Crit Care Med 177:433-439

10. Lynch DA, Godwin JD, Safrin S et al (2005) High-resolution computed tomography in idiopathic pulmonary fibrosis: diagnosis and prognosis. Am J Respir Crit Care Med 172:488-493

11. Chahal A, Sharif R, Watts J et al (2019) Predicting outcome in idiopathic pulmonary fibrosis: addition of fibrotic score at thinsection CT of the chest to gender, age, and physiology score improves the prediction model. Radiology Cardiothoracic Imaging 1: e180029

12. Ley B, Elicker BM, Hartman TE et al (2014) Idiopathic pulmonary fibrosis: CT and risk of death. Radiology 273:570-579

13. Watadani $T$, Sakai F, Johkoh $T$ et al (2013) Interobserver variability in the CT assessment of honeycombing in the lungs. Radiology 266:936-944

14. Bartholmai BJ, Raghunath S, Karwoski RA et al (2013) Quantitative CT imaging of interstitial lung diseases. J Thorac Imaging 28:298-307

15. Wu X, Kim GH, Salisbury ML et al (2019) Computed tomographic biomarkers in idiopathic pulmonary fibrosis. The future of quantitative analysis. Am J Respir Crit Care Med 199:12-21

16. Uppaluri R, Hoffman EA, Sonka M, Hartley PG, Hunninghake GW, McLennan G (1999) Computer recognition of regional lung disease patterns. Am J Respir Crit Care Med 160:648-654

17. Salisbury ML, Lynch DA, Van Beek EJ et al (2017) Idiopathic pulmonary fibrosis: the association between the adaptive multiple features method and fibrosis outcomes. Am J Respir Crit Care Med 195:921-929

18. Jacob J, Bartholmai BJ, Rajagopalan S et al (2016) Automated quantitative computed tomography versus visual computed tomography scoring in idiopathic pulmonary fibrosis. J Thorac Imaging 31:304-311

19. Maldonado F, Moua T, Rajagopalan S et al (2014) Automated quantification of radiological patterns predicts survival in idiopathic pulmonary fibrosis. Eur Respir J 43:204-212

20. Jacob J, Bartholmai BJ, Rajagopalan S et al (2017) Mortality prediction in idiopathic pulmonary fibrosis: evaluation of computer- 
based CT analysis with conventional severity measures. Eur Respir J 49:1601011

21. Jacob J, Bartholmai BJ, Rajagopalan S et al (2018) Serial automated quantitative $\mathrm{CT}$ analysis in idiopathic pulmonary fibrosis: functional correlations and comparison with changes in visual CT scores. Eur Radiol 28:1318-1327

22. Nishimura K, Kitaichi M, Izumi T, Nagai S, Kanaoka M, Itoh H (1992) Usual interstitial pneumonia: histologic correlation with high-resolution CT. Radiology 182:337-342

23. Smith M, Dalurzo M, Panse P, Parish J, Leslie K (2013) Usual interstitial pneumonia-pattern fibrosis in surgical lung biopsies. Clinical, radiological and histopathological clues to aetiology. $\mathrm{J}$ Clin Pathol 66:896-903

24. Chiu B, Beletsky V, Spence JD, Parraga G, Fenster A (2009) Analysis of carotid lumen surface morphology using threedimensional ultrasound imaging. Phys Med Biol 54:1149

25. Pienaar R, Fischl B, Caviness V, Makris N, Grant PE (2008) A methodology for analyzing curvature in the developing brain from preterm to adult. Int J Imaging Syst Technol 18:42-68

26. Raghu G, Collard HR, Egan JJ et al (2011) An official ATS/ERS/ JRS/ALAT statement: idiopathic pulmonary fibrosis: evidencebased guidelines for diagnosis and management. Am J Respir Crit Care Med 183:788-824

27. Kim H, Yoon SH, Lee H-J et al (2020) Right-angled traction bronchiectasis in differentiating idiopathic pulmonary fibrosis without honeycombing from idiopathic nonspecific interstitial pneumonia. Invest Radiol 55:387-395

28. Hu S, Hoffman EA, Reinhardt JM (2001) Automatic lung segmentation for accurate quantitation of volumetric X-ray CT images. IEEE Trans Med Imaging 20:490-498

29. Lorensen WE, Cline HE (1987) Marching cubes: a high resolution 3D surface construction algorithm. Comput Graph (ACM) 21:163169
30. Park J, Jung J, Yoon SH, Goo JM, Hong H, Yoon J-H (2018) Inspiratory lung expansion in patients with interstitial lung disease: CT histogram analyses. Sci Rep 8:15265

31. Zhang B, Tian J, Dong D et al (2017) Radiomics features of multiparametric MRI as novel prognostic factors in advanced nasopharyngeal carcinoma. Clin Cancer Res 23:4259-4269

32. Harrell FE Jr (2015) Regression modeling strategies: with applications to linear models, logistic and ordinal regression, and survival analysis, 2nd edn. Springer, Berlin Heidelberg New York

33. Iwasawa T, Takemura T, Okudera K et al (2017) The importance of subpleural fibrosis in the prognosis of patients with idiopathic interstitial pneumonias. Eur J Radiol 90:106-113

34. Graham BL, Brusasco V, Burgos F et al (2017) 2017 ERS/ATS standards for single-breath carbon monoxide uptake in the lung. Eur Respir J 49:1600016

35. Drummond MB, Schwartz PF, Duggan WT et al (2008) Intersession variability in single-breath diffusing capacity in diabetics without overt lung disease. Am J Respir Crit Care Med 178: 225-232

36. Meredith C. McCormack DAK (2020) Pulmonary function laboratories: advice regarding COVID-19. Available via https://www. thoracic.org/professionals/clinical-resources/disease-relatedresources/pulmonary-function-laboratories.php. Accessed 17/10/ 2020

37. Heussel C, Kappes J, Hantusch R et al (2010) Contrast enhanced $\mathrm{CT}$-scans are not comparable to non-enhanced scans in emphysema quantification. Eur J Radiol 74:473-478

Publisher's note Springer Nature remains neutral with regard to jurisdictional claims in published maps and institutional affiliations. 